\title{
LA INCIDENCIA DE LA FORMACIÓN MATEMÁTICA EN EL PENSAMIENTO PROBABILÍSTICO DE LOS ESTUDIANTES
}

\author{
Amable Moreno \\ Universidad Tecnológica Nacional, Regional Mendoza, Argentina \\ amable.moreno@frm.utn.edu.ar
}

Entre los estudiantes para profesor de Matemática, de la provincia de Mendoza, Argentina; hemos encontrado cuatro tendencias de pensamiento probabilistico: Determinista, Personalista, Incertidumbre y Contingente (Moreno \& Cardeñoso,2014a, 2014b; Moreno, Cardeñoso \& González-García, 2014c, 2014d). Sin embargo, no sabemos especificamente cuáles son las tendencias que poseen los estudiantes que se encuentran concluyendo su formación académica y que en un plazo muy corto de tiempo ejercerán la docencia. Por esta razón, hemos realizado un estudio con los ochenta y ocho estudiantes que se encuentran en esta situación, con el fin de determinar la incidencia de la formación matemática en el pensamiento probabilístico. Las respuestas de los estudiantes a un cuestionario revelaron la presencia de tres tendencias de pensamiento probabilístico: Determinista, Causalidad e Incertidumbre, que difieren de las encontradas en el conjunto de todos los estudiantes para profesor de Matemática.

\section{INTRODUCCIÓN}

Continuando con la línea de investigación iniciada por Azcárate (1995); continuada por Cardeñoso (2001) sobre las tendencias de pensamiento probabilístico de estudiantes para profesor de educación primaria en el primer caso y de profesores en activo en el segundo; hemos realizado un amplio estudio con los estudiantes para profesor de Biología ( Moreno, Cardeñoso \& GonzálezGarcía; 2012,2014e) y para profesor de Matemática (Moreno \& Cardeñoso, 2014a, 2014b; Moreno, Cardeñoso \& González-García, 2013, 2014c, 2014d), de la provincia de Mendoza, Argentina el que nos ha revelado la existencia de cuatro tendencias de pensamiento probabilístico: tendencia al determinismo, tendencia al personalismo, tendencia a la incertidumbre y tendencia a la contingencia. Sin embargo, sabemos que lo que ocurre a nivel global no siempre se reproduce a nivel local. Por este motivo, ante la inmediatez del ejercicio de la actividad docente de los estudiantes del último año de la carrera para Profesor de Matemática; nos propusimos estudiar sus tendencias de pensamiento probabilístico, debido a la ausencia del tratamiento de estas nociones en la escuela secundaria.

Entendemos que los estudiantes del último año de la carrera han construido esquemas cognitivos más elaborados que los estudiantes de cursos inferiores. Un esquema es en Psicología Cognitiva, una construcción perteneciente a la representación mental del conocimiento conceptual. El esquema cognitivo organiza las características asociadas al concepto en un todo integrado en la memoria, y es usado en un procesamiento cognitivo tal como recordar, reconocer, razonar y tomar decisiones. El esquema organiza características del fenómeno aleatorio en categorías: el mecanismo físico de la producción de resultados, las secuencias de resultados, la probabilidad, la frecuencia esperada, la variabilidad, la enumeración de los elementos del espacio muestral, las clases particulares de fenómenos aleatorios en distintos contextos y la variable aleatoria.

\section{MÉTODO}

Para llevar adelante nuestra investigación; utilizamos el método descriptivo. Las técnicas de recogida de datos y análisis de datos se enmarcan en el enfoque cuantitativo. De esta manera, la recogida de datos se concretó mediante la técnica de encuesta, utilizando como instrumento un cuestionario con 48 ítems.

El cuestionario utilizado fue tomado de Cardeñoso (2001) sobre el cual se realizaron algunas modificaciones para adaptarlo a nuestro contexto físico y cultural. Las categorías usadas por el autor mencionado para el diseño del cuestionario fueron las siguientes:

Causalidad: Argumentaciones que tienen como criterio de reconocimiento de la aleatoriedad explicaciones en función de los diversos factores causales o en la ausencia de posibilidad de su control.

In: M.A. Sorto (Ed.), Advances in statistics education: developments, experiences and assessments. Proceedings of the Satellite conference of the International Association for Statistical Education (IASE), July 2015, Rio de Janeiro, Brazil. 
Multiplicidad : Argumentaciones que tienen como criterio de reconocimiento de la aleatoriedad la existencia de múltiples posibilidades en el desarrollo del fenómeno.

Incertidumbre : Argumentaciones en las que se utiliza como criterio de reconocimiento de la aleatoriedad la propia imprevisibilidad del suceso, sin profundizar en su explicación o análisis.

Subjetiva : Argumentaciones en las que utiliza como criterio de reconocimiento de la aleatoriedad consideraciones referidas a la propia vivencia o creencia subjetiva.

Contingencia : Argumentaciones estimativas de cuantificación de la probabilidad basadas en la comparación entre los casos favorables y desfavorables de un suceso.

Laplaciana : Argumentaciones estimativas de cuantificación de la probabilidad basadas en la proporción entre los casos favorables y casos posibles del fenómeno.

Frecuencial : Argumentaciones estimativas de cuantificación de la probabilidad basadas en la lectura frecuencial del fenómeno o de la información aportada.

Equiprobabilidad : Argumentaciones estimativas de cuantificación de la probabilidad basadas en justificaciones desde la equiposibilidad entre los resultados del fenómeno.

Experiencial : Argumentaciones estimativas de cuantificación de la probabilidad basadas en criterios fruto de la experiencia personal.

Tabla 1 Las respuestas de los estudiantes se transformaron en las siguientes variables cuantitativas

ALEA11 Cantidad de sucesos reconocidos correctamente como aleatorios y
argumentados desde la Causalidad por el estudiante para profesor.

ALEA12 Cantidad de sucesos reconocidos correctamente como aleatorios y argumentados desde la Multiplicidad por el estudiante para profesor.

ALEA13 Cantidad de sucesos reconocidos correctamente como aleatorios y argumentados desde la Incertidumbre por el estudiante para profesor.

ALEA14 Cantidad de sucesos reconocidos correctamente como aleatorios y argumentados desde la Subjetividad por el estudiante para profesor.

ALEA21 Cantidad de sucesos reconocidos incorrectamente como no aleatorios desde la Causalidad por el estudiante para profesor.

ALEA22 Cantidad de sucesos reconocidos incorrectamente como no aleatorios desde la Multiplicidad por el estudiante para profesor.

Cantidad de sucesos reconocidos incorrectamente como no aleatorios desde la

ALEA23 Incertidumbre por el estudiante para profesor.

ALEA24 Cantidad de sucesos reconocidos incorrectamente como no aleatorios desde la Subjetividad por el estudiante para profesor.

PRO 5 Cantidad de sucesos argumentados desde la Contingencia en la estimación de la probabilidad por el estudiante para profesor.

PRO 6 Cantidad de sucesos argumentados desde la Laplaciana en la estimación de la probabilidad por el estudiante para profesor.

PRO 7 Cantidad de sucesos argumentados desde la Frecuencial en la estimación de la probabilidad por el estudiante para profesor.

PRO 8 Cantidad de sucesos argumentados desde la Equiprobabilidad en la estimación de la probabilidad por el estudiante para profesor.

PRO 9 Cantidad de sucesos argumentados desde la Experiencial en la estimación de la probabilidad por el estudiante para profesor.

Las respuestas de los estudiantes al cuestionario se transformaron en variables cuantitativas, como se indica en la Tabla 1; a las que se les aplicó técnicas multivariantes: análisis de clusters y análisis discriminante. El cuestionario fue completado por 583 estudiantes para profesor de Matemática, de los cuales $88(15,09 \%)$ se encontraban cursando el último año de la carrera, cuyas respuestas son analizadas en este trabajo. Los resultados mostraron la presencia de tres tendencias de pensamiento probabilístico. Estas tendencias difieren de las encontradas a nivel global, es decir, en el conjunto de todos los estudiantes, y son: Determinista, Causalidad e Incertidumbre; lo que nos indicaría cierta influencia de la formación matemática en el desarrollo del pensamiento probabilístico. 


\section{RESULTADOS}

La aplicación del método de clusters permitió encontrar tres tendencias de pensamiento probabilístico, que fue corroborado por el análisis discriminante, confirmando que el $94,3 \%$ de los estudiantes resultaron bien clasificados por el análisis de clusters.

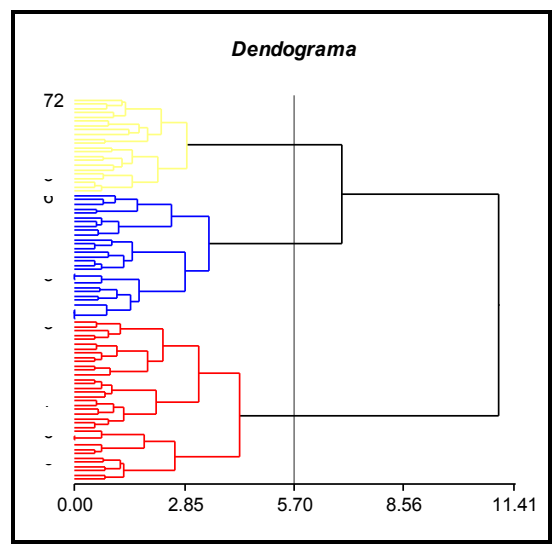

Figura 1. Dendograma de las tres tendencias de pensamiento probabilístico

La denominación de las tendencias de pensamiento probabilístico está determinada por las categorías dominantes en cada grupo. El grupo mayoritario es el grupo Determinista, que aglutina al 55,7\% de los estudiantes (49); seguido por el grupo Incertidumbre con el 28,4\% (25) de los estudiantes; y finalmente encontramos el grupo Causalidad con el 15,9\% (14). A continuación describimos las características fundamentales de cada grupo.

El grupo de estudiantes que representa la tendencia al Determinismo, es el que logra el menor reconocimiento de la aleatoriedad, en promedio, un estudiante de este grupo reconoce correctamente sólo cuatro sucesos como aleatorios de los doce propuestos. Cuando niegan la aleatoriedad lo hacen desde la Causalidad (ALEA21), desde la multiplicidad (ALEA 22) y desde la incertidumbre (ALEA23); negar la presencia de la aleatoriedad desde estos argumentos dan muestras acerca del desconocimiento del significado del término aleatorio. En relación con la estimación de la probabilidad, la categoría Frecuencial (PRO 7) logra su valor máximo, siendo la Equiprobabilidad (PRO 8) la categoría que alcanza su valor mínimo en este grupo. Por lo que podemos concluir que basan la estimación de la probabilidad sólo en la lectura frecuencial del fenómeno y en informaciones que aparecen en distintos medios.

El grupo de estudiantes que representa la tendencia a la Causalidad, es el segundo grupo en el reconocimiento de la aleatoriedad, en promedio, un estudiante de este grupo reconoce nueve sucesos como aleatorios de los doce propuestos. Se destaca por argumentar la aleatoriedad desde la Causalidad (ALEA11) y desde la Multiplicidad (ALEA12); por otra parte la negación incorrecta de la aleatoriedad la argumenta desde la Causalidad (ALEA21), categoría que alcanza el valor mínimo en este grupo y desde la Incertidumbre (ALEA23). En relación con la estimación de la probabilidad, la categoría más usada es la Equiprobabilidad (PRO8), la que representa un verdadero sesgo debido a que la mayoría de los fenómenos propuestos no están asociados a espacios muestrales equiprobables; por otra parte las categorías Laplaciana (PRO6) y Frecuencial (PRO 7) alcanzan valores mínimos. 
El grupo de estudiantes que representa la tendencia a la Incertidumbre, se destaca por el alto reconocimiento de la aleatoriedad; en promedio, un estudiante reconoce como aleatorio diez de los doce sucesos propuestos; es el que más usa la Incertidumbre (ALEA 13) en el reconocimiento de la aleatoriedad; mientras que la negación incorrecta de la aleatoriedad la hacen desde la Multiplicidad (ALEA22) y desde la Incertidumbre (ALEA23), categorías que alcanzan valores mínimos en este grupo. Cuando estiman la probabilidad, se destaca el uso de la argumentación Laplaciana (PRO 6).

En la Figura 2 se muestran los valores medios de las variables consideradas en cada una de las tres tendencias de pensamiento probabilístico de los estudiantes del último año del Profesorado de Matemática.

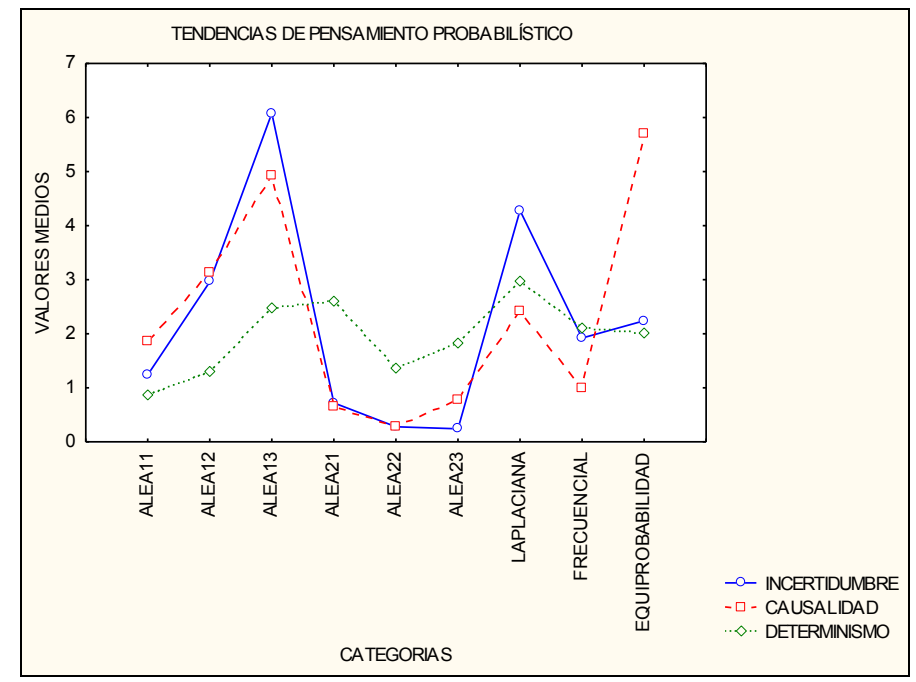

Figura 2. Valores medios de las variables en cada una de las tres tendencias de pensamiento probabilístico

\section{DISCUSIÓN}

Si comparamos el grupo Determinista de los estudiantes del último año de la carrera, con la misma tendencia encontrada en el grupo de todos los estudiantes (Moreno \& Cardeñoso, 2014a, 2014b); comprobamos que se destaca en los primeros un mayor uso de la Incertidumbre (ALEA13) y un menor uso de la Causalidad (ALEA11) en el reconocimiento de la aleatoriedad, mientras que niegan incorrectamente la aleatoriedad desde la Causalidad (ALEA21) con mayor frecuencia; y en relación a la estimación de la probabilidad argumentan mayoritariamente desde la categoría Laplaciana (PRO 6), seguida por la categoría Frecuencial (PRO 7), destacándose un menor uso de la Equiprobabilidad (PRO8).

Comparando la tendencia a la Incertidumbre entre los estudiantes del último año de la carrera con la misma tendencia encontrada en el grupo de todos los estudiantes, los primeros hacen un mayor uso de la categoría Multiplicidad (ALEA12) y un igual uso de la Incertidumbre (ALEA 13), mientras que usan en menor medida la categoría Causalidad (ALEA11) en el reconocimiento de la aleatoriedad y la categoría Incertidumbre (ALEA23) en la negación incorrecta de la aleatoriedad. En relación a la estimación de la probabilidad aplican mayoritariamente la categoría Laplaciana (PRO 6) y en menor medida la Equiprobabilidad (PRO 8).

La comparación de las tendencias al Determinismo y a la Incertidumbre detectadas en los estudiantes del último año de la carrera con las encontradas en el grupo de todos los estudiantes, revelan una ligera evolución. Además, aparece una nueva tendencia a la Causalidad, ausente a nivel global, o sea en el conjunto de todos los estudiantes; que si bien tiene un alto reconocimiento de la aleatoriedad, en muchos casos utilizan argumentos causales para justificar la aleatoriedad, desconociendo la componente aleatoria del fenómeno considerado, que es la que le otorga el carácter aleatorio; como ocurre en fenómenos del contexto cotidiano y del físico-natural. Además, 
se destaca una fuerte presencia del sesgo de equiprobabilidad, lo que indica la falta de concepciones normativas acerca de la estimación de la probabilidad.

Podemos concluir que el 55,7\% de estudiante que integran la tendencia al Determinismo y el $15,9 \%$ de los estudiantes que integran la tendencia a la Causalidad, conforman un grupo de estudiantes que están próximos a ejercer la docencia en el nivel de secundaria. Estos estudiantes poseen una formación parcial de las concepciones probabilísticas, que no les permitirán abordar con resultados satisfactorios la formación probabilística de los estudiantes del nivel de secundaria. Sólo el 28,4\% de los estudiantes estarían capacitados para abordar la enseñanza, desde un enfoque basado en fenómenos aleatorios del mundo lúdico, y en la estimación probabilística fundamentalmente desde la categoría laplaciana; la que implica la consideración de espacios muestrales finitos y equiprobables.

Estos resultados revelan la necesidad de una revisión del currículo de Probabilidad y Estadística en la formación del futuro profesor de Matemática.

Esperábamos que estos estudiantes, que han transitado cuatro niveles propedeúticos, presentaran esquemas cognitivos más evolucionados acerca de los distintos fenómenos aleatorios y la estimación probabilística.

A pesar de haber logrado un ligero avance en las tendencias de pensamiento probabilístico de los estudiantes del último año de la carrera, respecto del conjunto de todos los estudiantes, no es suficiente para afrontar la enseñanza de la probabilidad en el nivel de secundaria, y en este sentido sería oportuno seguir las sugerencias de Cardeñoso, Azcárate \& Serradó (2005); Batanero (2005); Gal (2005); Batanero, Arteaga, Ruiz \& Rao (2010).

Considerando los marcos teóricos sobre los cuales se apoyan los diseños curriculares, contemplan el enfoque clásico de la Estadística. En oposición a éstos, Krynski \& Tenenbaum (2007) en un estudio sobre razonamiento probabilístico bajo incertidumbre, concluyen que el formalismo de las redes bayesianas como modelo teórico del razonamiento bajo incertidumbre, puede explicar mejor el comportamiento de las personas ante juicios causales frente a las perspectivas frecuentistas. Para estos autores las desviaciones de los juicios de las personas respecto de las normas tradicionales, se puede explicar en términos de desajuste entre la Estadística que se les enseña a las personas y los modelos causales que intuitivamente construyen para afrontar el razonamiento probabilístico.

Por lo tanto, podríamos sugerir la incorporación a los diseños curriculares, de un marco teórico que complemente la normativa que aporta la Estadística Clásica; es decir, nos referimos a la Estadística Bayesiana; lo que podría generar modelos mentales que proporcionen interpretaciones más ajustadas al mundo real.

\section{REFERENCIAS}

Azcárate, P. (1995). El Conocimiento Profesional de los Profesores sobre las nociones de Aleatoriedad y Probabilidad. Su estudio en el caso de la educación Primaria (Tesis doctoral). Universidad de Cádiz, Cádiz.

Azcárate, P., \& Cardeñoso, J. M. (2003). Conocimiento profesional de referencia con relación al conocimiento probabilístico. Una aproximación a las ideas de los futuros profesores de primaria sobre el mismo. 27 Congreso Nacional de Estadística e Investigación Operativa Lleida.

Batanero, C. (2005). Significados de la probabilidad en la educación secundaria. Revista Latinoamericana de Investigación en Matemáticas, 8(3), 247-264.

Batanero, C., Arteaga, P., Ruiz, B., \& Rao, R. (2010). Assessing pre-service teachers conceptions of randomness through project work. In C. Reading (Ed.) Proceedings of the Eight International Conference for Teaching Statistics. Lbjubjana: IASE. Recuperado de https://www.stat.auckland.ac.nz/ iase/publications/icots8/ICOTS8_5A3_BATANERO.pdf.

Cardeñoso, J. M. (2001). Las creencias y conocimientos de los profesores de primaria andaluces sobre la Matemática escolar. Modelización de conceptos sobre la aleatoriedad y probabilidad. (Tesis doctoral). Universidad de Cádiz, Cádiz.

Cardeñoso, J. M., Azcárate, P., \& Serradó, A. (2005). Los obstáculos en el aprendizaje del conocimiento probabilístico: su incidencia desde los libros de texto. Statistics Education Research Journal, 4(2), 59-81. 
Gal, I. (2005). Towards "probability literacy" for all citizens: Building blocks and instructional dilemmas. En G. Jones (Ed.), Exploring probability in school: Challenges for teaching and learning, 39-63. New York: Springer.

Krynski, T.R., \& Tenenbaum, J.B. (2007). The Role of Causality in Judgment Under Uncertainty. Journal of Experimental Psychology: General, 136(3), 430-450. Recuperado de: http://web.mit.edu/cocosci/Papers/krynski-tenenbaum-jepgen07.pdf.

Moreno, A., Cardeñoso, J.M., \& González-García, F. (2012). Un estudio exploratorio de las tendencias de pensamiento probabilístico de los estudiantes del profesorado de biología. En A. Estepa; Á. Contreras; J. Deulofeu; M. C. Penalva; F. J. García y L. Ordóñez (Eds.), Actas Investigación en Educación Matemática XVI, 407-415.

Moreno, A., Cardeñoso, J. M., \& González-García, F. (2013). La aleatoriedad desde la perspectiva de los estudiantes del Profesorado de Matemática. En J.M. Contreras, G.R. Cañadas, M.M. Gea y P. Arteaga (Eds.) Actas de las I Jornadas Virtuales en Didáctica de la Estadística, Probabilidad y Combinatoria. Granada: Departamento Didáctica Matemática de la Universidad de Granada, 367-372. Recuperado de: http://www.jvdiesproyco.es/documentos/ACTAS/Actas\%20jornadas.pdf

Moreno, A., \& Cardeñoso, J. M. (2014a). "Overview of Prospective Mathematics Teachers' Probabilistic Thinking”. En K. Makar, B. De Sousa \& R. Gould (Eds.), ICOTS-9 Conference Proceedings. Sustainability in statistics education. $9^{\text {th }}$ International Conference on Teaching Statistics. Flagstaff, Arizona. Recuperado de: http://icots.info/9/proceedings/pdfs/ICOTS9 C262 MORENO.pdf

Moreno, A., \& Cardeñoso, J. M. (2014b). Alfabetización Probabilística: Un reto para los profesores de secundaria. Actas del Congreso Iberoamericano de Ciencia, Tecnología, Innovación y Educación. ISBN 978-84-7666-210-6. Documento 203. Buenos Aires. Argentina.

Moreno, A., Cardeñoso, J. M., \& González-García, F. (2014c). La Aleatoriedad en los Profesores de Biología y de Matemática en Formación: Análisis y Contraste de Significados. Revista Eureka sobre Enseñanza y Divulgación de las Ciencias, 11(2), 198-215.

Moreno, A., Cardeñoso, J. M., \& González-García, F. (2014d). Los significados de la probabilidad en los profesores de matemática en formación: un análisis desde la teoría de los modelos mentales. En Actas de la XXVIII Reunión Latinoamericana de Matemática Educativa. Barranquilla. Colombia.

Moreno, A.; Cardeñoso, J. M., \& González-García, F. (2014e). El Pensamiento Probabilístico de los Profesores de Biología en Formación. Bolema: Boletim de Educação Matemática, 28(50), 1415-1442. Recuperado de:

http://www.periodicos.rc.biblioteca.unesp.br/index.php/bolema/article/view/7338/6503 